\title{
Performance Analysis of DWDM System Having 0.8- Tbps Date Rate with 80 Channels
}

Sahil Kakalia and Munish Singh

Department of Electronics and Communication, Lovely Professional University, Phagwara-144411, Punjab, India; sahilkakalia@gmail.com, munish.17486@|pu.co.in

\begin{abstract}
Objectives: The main aim of this research paper is to design a 80 channel DWDM system with each channel having $10 \mathrm{Gbps}$ data rate multiplexed with frequency spacing $100 \mathrm{GHz}$. Methods/Statistical analysis: Dispersion is an important factor to be considered while designing a DWDM system. Dispersion affects the penalties due to various types of fiber nonlinearities. Single mode fiber is preferred for long distance communication over Multimode fiber. In this proposed work Optisystem 7.0 simulator is used to analyze dispersion effect. The system performance is optimized by using Dispersion compensation fiber to compensate for the dispersion produced by single mode fiber. Findings: The system performance is limited by the dispersion. In order to compensate this we have used Dispersion compensation fiber. Between amplifier spans is standard single-mode fiber, but at each amplifier location, dispersion compensating fiber having a negative chromatic dispersion is introduced. By using this we have successfully designed a DWDM system with 80 channels each 10 Gbps data rate multiplexed with frequency spacing $100 \mathrm{GHz}$. Application/Improvements: It is realized that, in coming future, DWDM can emerge as a promising technique to increase the capacity and meet the bandwidth requirement. This work can further be extended to more number of channels i.e. 100 channels or more with even smaller frequency spacing.
\end{abstract}

Keywords: Dispersion Compensating Fiber (DCF), Bit Error Rate (BER), Dense Wavelength Division Multiplexing (DWDM)

\section{Introduction}

In the recent years, the growth in the internet activities like E-mail, audio-video conferencing, multimedia services, has increased greatly. There has been a great demand in the increase in data rate due to increase of use of large bandwidth applications and internet, thereby putting a lot of pressure on TDM to stretch its limits. It has become quite clear that as we are approaching $21^{\text {st }}$ century, our society will be inhabited by the need of information services. In the past, communication only meant voice calls. But now the entire definition of communication has changed. Communication now corresponds to not only a demand of high quality voice but also video calling, text messaging, transfer of data files, videos and a lot more. Every human activity now depends on reliable and rapid communication networks ${ }^{5}$. This has led to an increase in demand for higher data rates for high speed internet services ${ }^{1}$. In order to meet these increasing data rate, the increase in bandwidth is the only solution. The bandwidth can be increased in by installing more number of cables, increasing system bit rate to multiplex more signals or multiplex different wavelengths (DWDM).

In order to meet this growing demand of bandwidth, a technique which combines various wavelengths together called as Dense Wavelength Division Multiplexing (DWDM) is developed. It is used to increase fiber's capacity $\underline{2}$.

It is a technique allowing multiple wavelengths to be transmitted simultaneously over a single fiber, thereby allowing carriers to increase the data rate by using already laid single fiber. Each information stream is transmitted on a unique wavelength. All the wavelengths are combined by using a mux ${ }^{15}$. DWDM uses an optical signal

${ }^{*}$ Author for correspondence 
which carries multiple wavelengths carrying user data on each wavelength. DWDM system can handle more number of users per wavelength. But dispersion compensation plays a key role in DWDM at bit rate greater than $10 \mathrm{Gbps}$. It can be compensated by using dispersion compensating fiber ${ }^{3}$. WDM systems suffer from four wave mixing effect. Due to this reason, non-zero dispersion-shifted fibers are preferred. Four wave mixing effect can be minimized using OPC and dispersion compensating fibers ${ }^{\underline{13}}$.EDFA's are used in addition to DCF's to amplify and regenerate the optical signal. The mix- compensation performance is the best ${ }^{8}$.

For long distances, Erbium-Doped Fiber Amplifier (EDFA) is preferred ${ }^{6}$. If EDFA is placed before SMF, it is called pre compensation and if it is used after SMF, it is called post compensation. Both these techniques can be combined to produce and deliver a good quality of optical signal at the transmitter ${ }^{\mathrm{T}}$. The noise figure and gain depends on fiber length for an EDFA ${ }^{11}$.

Fiber Bragg grating (FBG) is playing significant role in optical fiber communication as filter, stabilizer, gain flattening filter, dispersion compensator, optical router etc ${ }^{14}$. Furthermore, it is also used as sensor for sensing temperature, pressure and strain etc. The FBG is a special form of optical fiber where the refractive index of the core is variable $\frac{12}{2}$. As a result, the wavelength response of the fiber changes and various applications emerge ${ }^{10}$.

\section{Simulation Setup}

Figure 1 shows the simulation setup of an 80 channel DWDM setup. In this set up, at the transmitter side we have used WDM transmitter and WDM mux. The Bit rate used is $10 \mathrm{~Gb} / \mathrm{s}$. The channel consists of Optical fiber, loop control, dispersion compensating fibers and EDFA's. The optical channel has two optical fibers of $25 \mathrm{~km}$ length, so a total fiber of $50 \mathrm{~km}$ is used. Various EDFA's are used to improve the quality of the signal. In addition, Dispersion compensating fiber is also used to tackle the dispersion on the channel. The receiver side consists of WDM demux, optical receivers and BER analyzers for the analysis of WDM link. This entire set up is implemented and analyzed using "Optisystem 7.0".

\subsection{Transmitter}

A transmitter is that component of a DWDM system which generates the different wavelengths for different channels and multiplexes them or combines them on to a single fiber. The WDM transmitter here generates 80 different wavelengths for 80 different channels with frequency spacing $100 \mathrm{GHz}$. The optical signal is transmitted at a frequency of $1555 \mathrm{~nm}$ with power $5 \mathrm{dbm}$ with NRZ type of modulation. These 80 different wavelengths are multiplexed using WDM mux. The various parameters used at the transmitter side are as shown below and are taken from simulation on optisystem software.

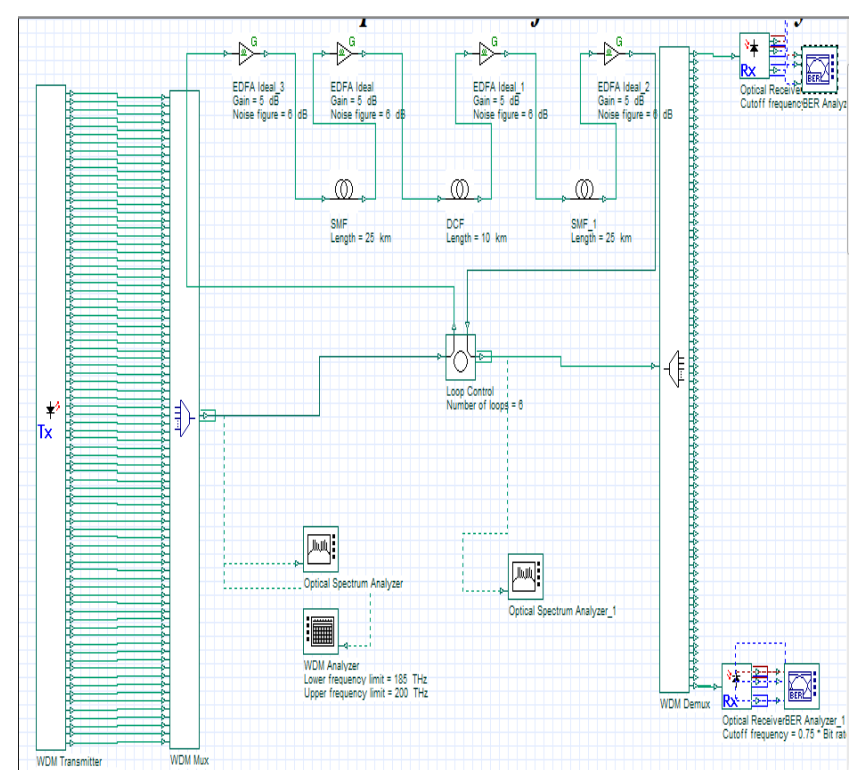

Figure 1. Structure of an 80 channel DWDM system.

Table 1. Simulation Parameters of WDM Transmitter

\begin{tabular}{|l|l|}
\hline Parameters & Value \\
\hline Frequency & $1555 \mathrm{~nm}$ \\
\hline Frequency spacing & $100 \mathrm{GHz}$ \\
\hline Power & $5 \mathrm{dBm}$ \\
\hline Extinction ratio & $30 \mathrm{~dB}$ \\
\hline Modulation & $\mathrm{NRZ}$ \\
\hline
\end{tabular}

\subsection{Optical Channel}

The multiplexed optical signal consisting of all the 80 wavelengths is transmitted over the channel. The optical channel consists of two single mode fibers of $25 \mathrm{~km}$ length so the total length is $50 \mathrm{~km}$. We have used Dispersion compensating fiber to counter the dispersion occurring in the system. The optical signal is amplified at various intervals of distance by using EDFA's. For this various EDFA's and DCF are used in such an arrangement that a 
SMF have an EDFA both in front and back. The various parameters of Optical channel used are as shown below:

Table 2. Simulation parameters of EDFA

\begin{tabular}{|l|l|}
\hline Parameters & Value \\
\hline Operation mode & Gain control \\
\hline Gain & $5 \mathrm{~dB}$ \\
\hline Power & $10 \mathrm{dBm}$ \\
\hline Noise figure & $6 \mathrm{~dB}$ \\
\hline
\end{tabular}

Table 3. Simulation parameters of SMF and DCF

\begin{tabular}{|l|l|}
\hline Parameters & Value \\
\hline Length & $25 \mathrm{~km}$ \\
\hline Attenuation & $0.2 \mathrm{~dB} / \mathrm{km}$ \\
\hline DCF length & $10 \mathrm{~km}$ \\
\hline Attenuation & $\mathrm{dB} / \mathrm{km}$ \\
\hline
\end{tabular}

\subsection{Receiver}

The receiver consists of WDM de-mux which de-multiplexes or separates the multiplexed signal into its constituent wavelengths. In addition to de-mux, the receiver also has optical receiver and a BER analyzer. The BER analyzer is used to analyze the various parameters of WDM link such as BER, Q factor, eye height etc. Various parameters used at receiver in optisystem software are as shown below:

Table 4. Simulation parameters of WDM de-mux and optical receiver

\begin{tabular}{|l|l|}
\hline Parameters & Value \\
\hline Bandwidth & $80 \mathrm{GHz}$ \\
\hline Responsivity & $1 \mathrm{~A} / \mathrm{W}$ \\
\hline Cut off frequency & $0.75^{\star}$ bit rate \\
\hline
\end{tabular}

\section{Result and Discussion}

By using the dispersion compensating fiber as dispersion compensator, we have optimized and established an 80 channel DWDM link. The min. BER is found to be $1.24865 \mathrm{e}-087$ and Q factor is found to be 19.8088 on channel 1 as shown in Figure. 2. The eye diagram in fig. 3 shows the Q factor to be 14.5085 and BER to be $5.33659 \mathrm{e}-$ 048 . The eye diagram and other parameters for channel no. $1,8,16,24,32,40,48,56,64,72,80$ are analyzed and as shown from Figure. 2 to 12. A summarized table is also given at the end to show the results. All these figures and tables are the result obtained from optisystem simulator.

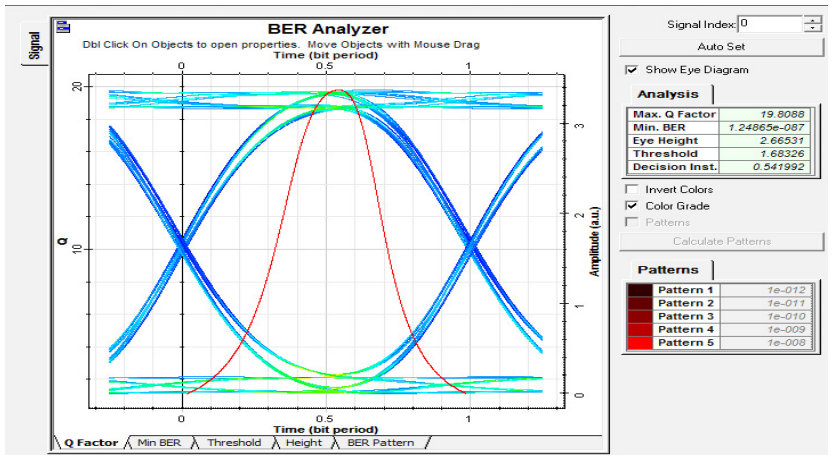

Figure 2. Eye diagram analysis of first channel.

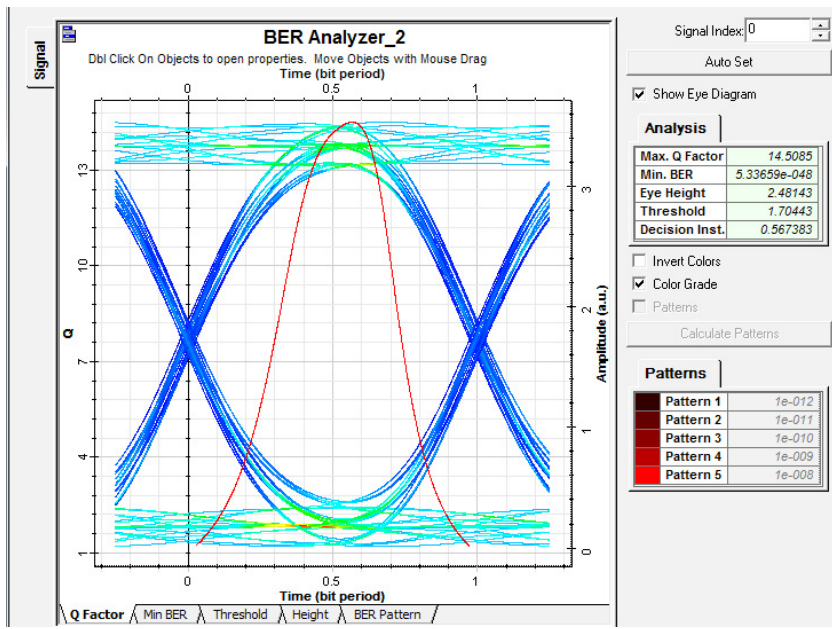

Figure 3. Eye diagram analysis of eighth channel.

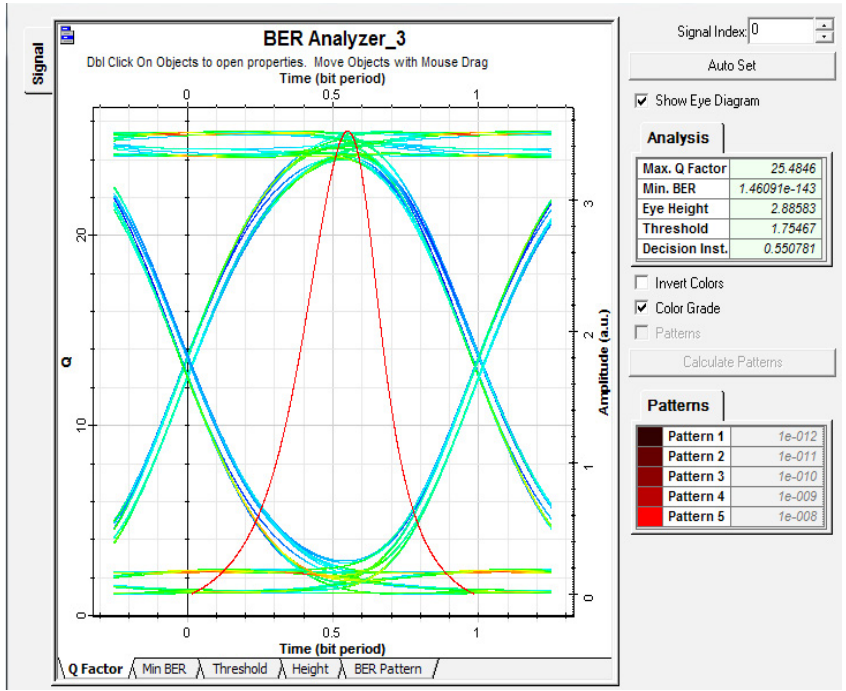

Figure. 4. Eye diagram analysis of sixteenth channel. 


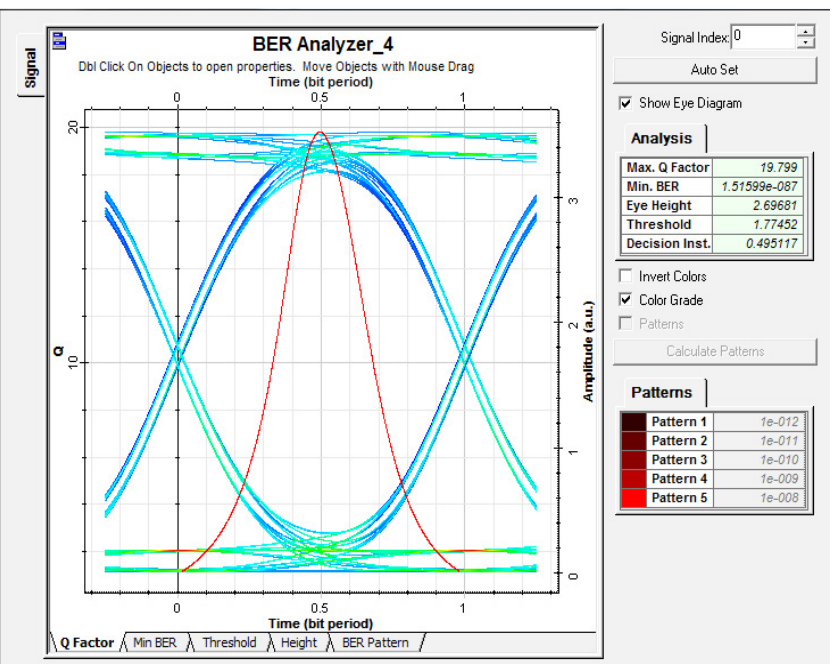

Figure 5. Eye diagram analysis of twenty fourth channel.

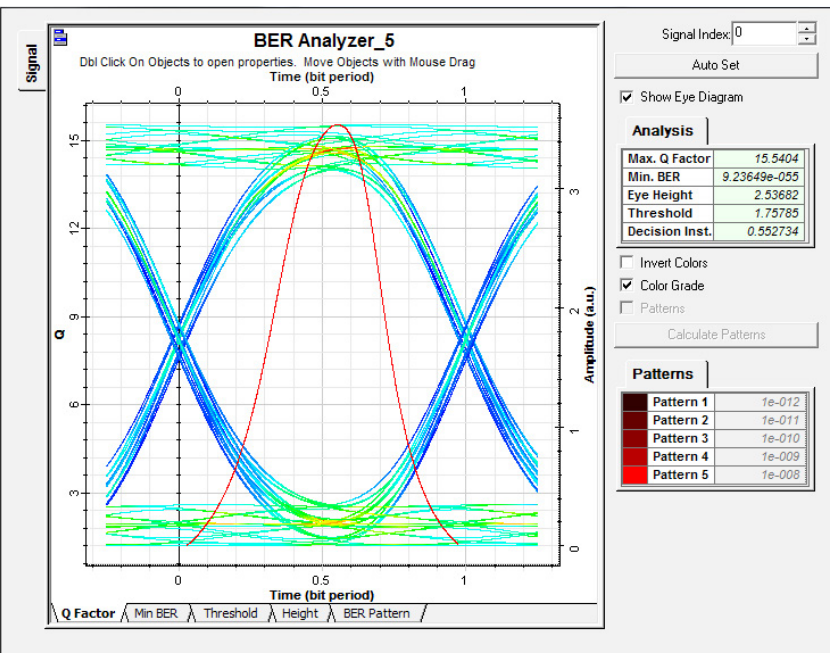

Figure 6. Eye diagram analysis of thirty second channel.

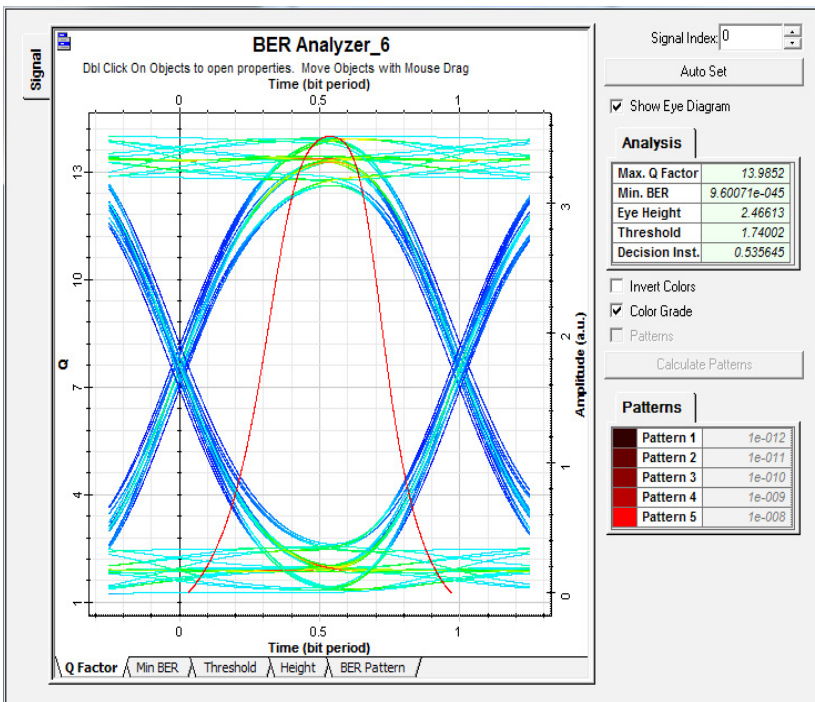

Figure 7. Eye diagram analysis of fourth channel.

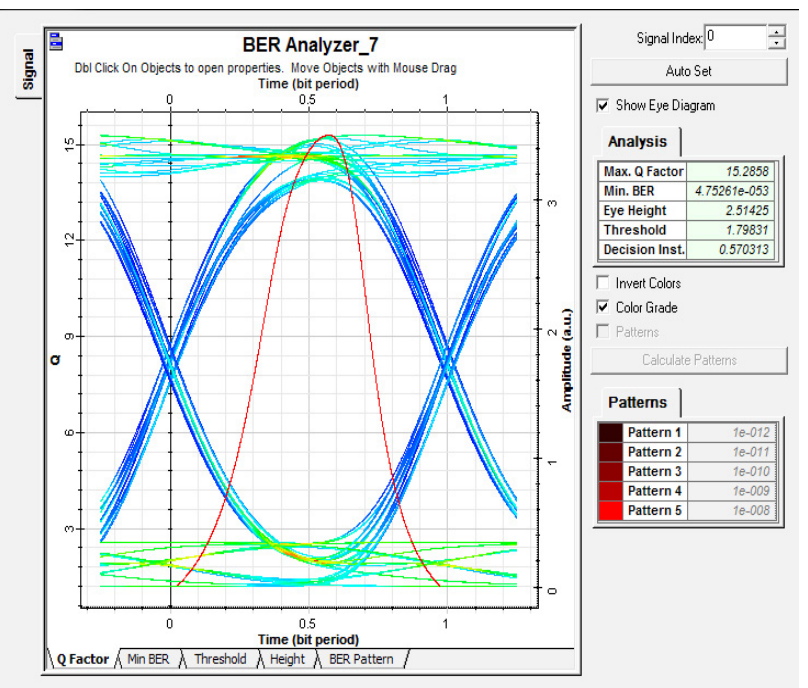

Figure 8. Eye diagram analysis of forty eighth channel.

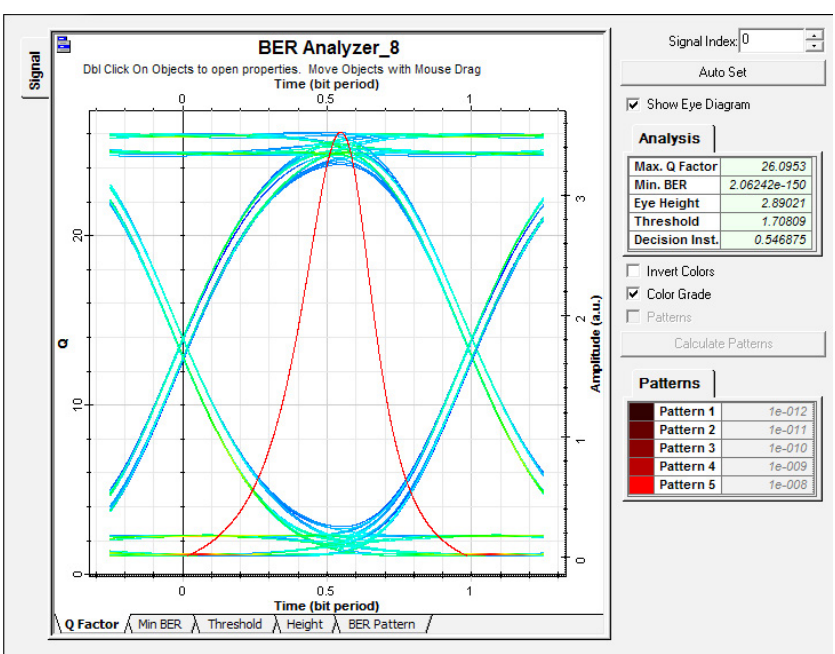

Figure 9. Eye diagram analysis of fifty sixth channel.

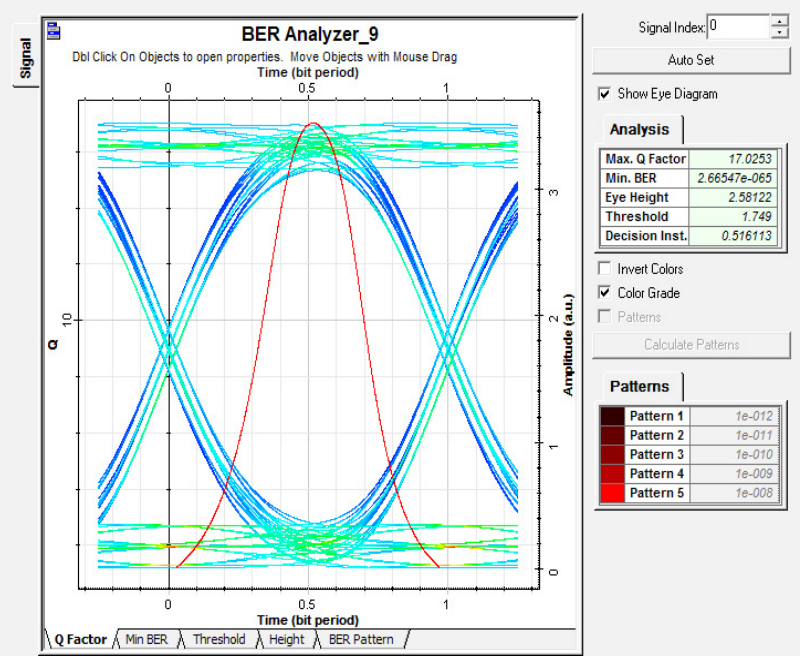

Figure 10. Eye diagram analysis of sixty fourth channel. 


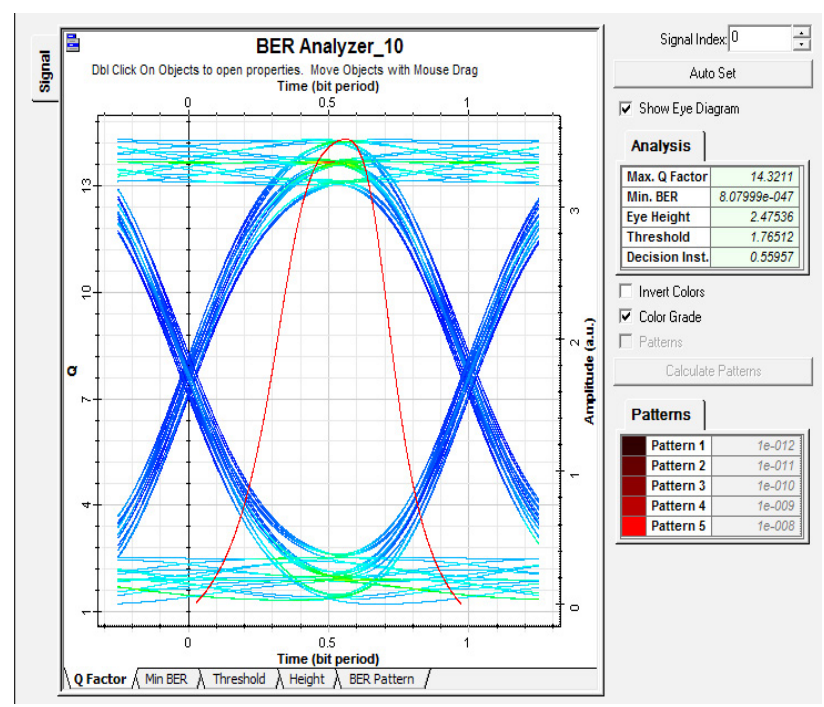

Figure 11. Eye diagram analysis of seventy second channel.

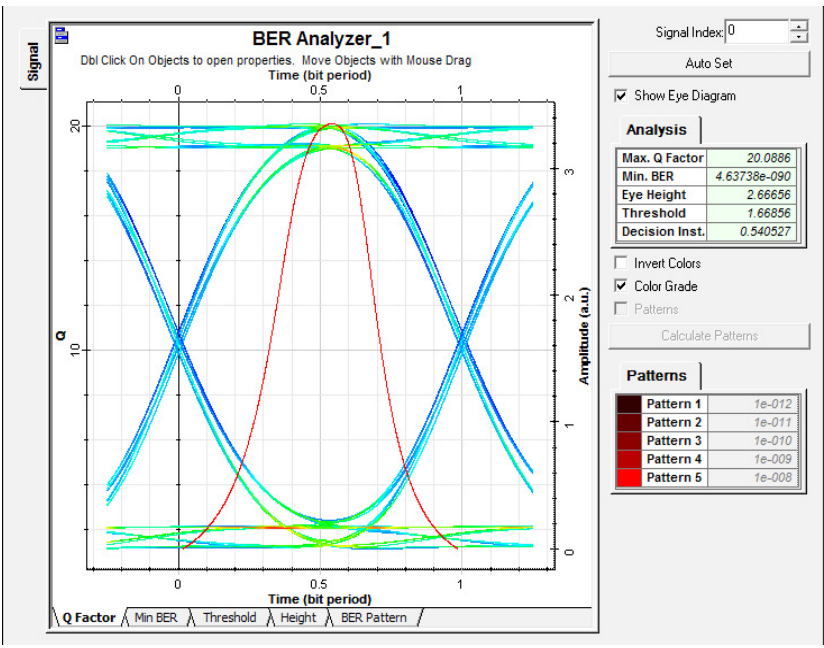

Figure 12. Eye diagram analysis of eightieth channel.

Table 5. Values of q factor and BER for various channels

\begin{tabular}{|l|l|l|}
\hline Channel no. & Max. Q factor & Min. BER \\
\hline 1 & 19.808 & $1.24865 \mathrm{e}-087$ \\
\hline 8 & 14.5085 & $5.33659 \mathrm{e}-048$ \\
\hline 16 & 25.4846 & $1.46091 \mathrm{e}-143$ \\
\hline 24 & 19.799 & $1.51599 \mathrm{e}-087$ \\
\hline 32 & 15.504 & $9.23649 \mathrm{e}-055$ \\
\hline 40 & 13.9852 & $9.60071 \mathrm{e}-045$ \\
\hline 48 & 15.2858 & $4.75261 \mathrm{e}-053$ \\
\hline 56 & 26.0953 & $2.06242 \mathrm{e}-150$ \\
\hline 64 & 17.0253 & $2.66547 \mathrm{e}-065$ \\
\hline 72 & 14.3211 & $8.07999 \mathrm{e}-047$ \\
\hline 80 & 20.0886 & $4.63738 \mathrm{e}-090$ \\
\hline
\end{tabular}

\section{Conclusion}

In this work, an optimized DWDM link using DCF for dispersion compensation is presented. The system performance is limited by the dispersion. In order to compensate this we have used Dispersion compensation fiber. Between amplifier spans is standard single-mode fiber, but at each amplifier location, dispersion compensating fiber having a negative chromatic dispersion is introduced. By using this we have successfully designed a DWDM system with 80 channels each 10 Gbps data rate multiplexed with frequency spacing $100 \mathrm{GHz}$. Various EDFA's are also used to amplify and regenerate the signal. This work can be further extended to 160 channels in future as well.

\section{References}

1. Analysys Mason. Fibre Capacity Limitations in Access Networks. Report for OFCOM, Jan. 2010.

2. CIENA Corporation. Dense Wavelength Division Multiplexing. ATG's Communications and Networking Technology Guide Series.

3. Manpreet Kaur, Himali Sarangal. Simulative Investigation

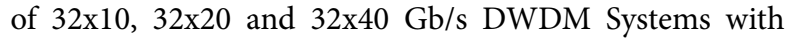
Dispersion Compensating Fibers. International Journal of Signal Processing, Image Processing and Pattern Recognition. 2015; 8:127-34.

4. Tharwat, Marwa M, Islam Ashry, Ali Elrashidi, Amr M. Mahros. Study of green wavelength-division multiplexed optical communication systems using cascaded fiber Bragg grating. Optical Fiber Technology. 2014; 20(5):467-72.

5. Karan Gupta, Taranand Mukhopadhyay, Abhishek Goyanka. Design and simulation of a chirped Fiber Bragg Grating based demultiplexer for ultra-dense Wavelength Division Multiplexing based Passive Optical Networks. IEEE International Conference on Advanced Networks and Telecommuncations Systems. 2014. p. 1-5.

6. Almalaq, Yasser, Mohammad Matin. Analysis of Transmitting 40Gb/s CWDM Based on Extinction Value and Fiber Length Using EDFA. America, University of Denver. 2014.

7. Gaurav Soni, Rupinderjit Kaur. Performance analysis of WDM link using Dispersion Compensating Fiber at different wavelengths. IEEE International Conference on Contemporary Computing and Informatics. 2014.p. 1240_44.

8. Wu, Qiang, Chong-xiu Yu, Yu Xin, De-qi YAO, Xiang-jun XIN, Yuehui HU, Yue-fei WANG. Analysis on dispersion compensation with DCF. Semiconductor OptoelectronicsChongqing. 2013; 24:186-88. 
9. Simranjit Singh, Kaler RS. Flat-gain L-band Raman-EDFA hybrid optical amplifier for dense wavelength division multiplexed system. IEEE Photonics Technology Letters. 2013; 25(3):250-52.

10. Faiyaz, Naqib Muhammad, Asif Iftekhar Omi, Mohammad Faisal. Optimization of apodization profile of chirped fiber Bragg grating for chromatic dispersion compensation, Dispersion compensation using chirped apodized FBG. IEEE International Conference on Electrical Engineering and Information and Communication Technology. 2014.p. $1-5$.

11. Pain Somnath, Ricky Anthony, Sanchita Goswami, Debolina Brahma, Sambhunath Biswas. Analysis of Length Dependent Gain and Noise Figure in a Dual Stage L-Band EDFA configuration. IEEE International Conference on Electronics, Communication and Instrumentation. 2014. p. $1-4$.
12. Gerd, Keiser. Optical fiber communication, NY, McGrawHil, Fourth Edition, 2008.p.83-105.

13. Manisha Ajmani, Preeti Singh. Comparative Analysis of DCF and OPC as Means to Minimize FWM in WDM System. Indian Journal of Science and Technology. 2015; 8(27):1-12.

14. Ramandeep Kaursidhu, Harbinder Singh. Suppression of FWM Effects by using Cost Effective Combined DCF and FBG Module. Indian Journal of Science and Technology. 2016; 9(36):1-5.

15. Sana Rebhi, Monia Najjar, Rezig Houria. Performance Analysis of a $2 \mathrm{~Tb} / \mathrm{s}$ DWDM System Based on Narrow Channel Spacing and Multi- Diagonal Code. 22nd AsiaPacific Conference on Communications. 2016. p.1-5. 